\title{
Genome-wide characterization of copy number variations in the host genome in genetic resistance to Marek's disease using next generation sequencing
}

Hao Bai ${ }^{1,2,3}$ (D), Yanghua He ${ }^{2,4}$, Yi Ding ${ }^{2}$, Qin Chu ${ }^{2,5}$, Ling Lian' ${ }^{6}$ Eliyahu M. Heifetz ${ }^{7}$, Ning Yang ${ }^{6}$, Hans H. Cheng ${ }^{8}$, Huanmin Zhang ${ }^{8}$, Jilan Chen ${ }^{3}$ and Jiuzhou Song ${ }^{2 *}$

\begin{abstract}
Background: Marek's disease (MD) is a highly neoplastic disease primarily affecting chickens, and remains as a chronic infectious disease that threatens the poultry industry. Copy number variation (CNV) has been examined in many species and is recognized as a major source of genetic variation that directly contributes to phenotypic variation such as resistance to infectious diseases. Two highly inbred chicken lines, $\sigma_{3}$ (MD-resistant) and 72 (MD-susceptible), as well as their $F_{1}$ generation and six recombinant congenic strains (RCSs) with varied susceptibility to MD, are considered as ideal models to identify the complex mechanisms of genetic and molecular resistance to MD.
\end{abstract}

Results: In the present study, to unravel the potential genetic mechanisms underlying resistance to MD, we performed a genome-wide CNV detection using next generation sequencing on the inbred chicken lines with the assistance of CNVnator. As a result, a total of 1649 CNV regions (CNVRs) were successfully identified after merging all the nine datasets, of which 90 CNVRs were overlapped across all the chicken lines. Within these shared regions, 1360 harbored genes were identified. In addition, 55 and 44 CNVRs with 62 and 57 harbored genes were specifically identified in line $\sigma_{3}$ and $7_{2}$, respectively. Bioinformatics analysis showed that the nearby genes were significantly enriched in $36 \mathrm{GO}$ terms and 6 KEGG pathways including JAK/STAT signaling pathway. Ten CNVRs (nine deletions and one duplication) involved in 10 disease-related genes were selected for validation by using quantitative real-time PCR (qPCR), all of which were successfully confirmed. Finally, GPCR was also used to validate two deletion events in line $7_{2}$ that were definitely normal in line $6_{3}$. One high-confidence gene, IRF2 was identified as the most promising candidate gene underlying resistance and susceptibility to MD in view of its function and overlaps with data from previous study.

Conclusions: Our findings provide valuable insights for understanding the genetic mechanism of resistance to MD and the identified gene and pathway could be considered as the subject of further functional characterization.

Keywords: Copy number variation, Chicken, Inbred lines, Recombinant congenic strains, Marek's disease, Next generation sequencing

\footnotetext{
* Correspondence: songj88@umd.edu

${ }^{2}$ Department of Animal \& Avian Sciences, University of Maryland, College Park, MD 20742, USA

Full list of author information is available at the end of the article
}

C C The Author(s). 2020 Open Access This article is licensed under a Creative Commons Attribution 4.0 International License, which permits use, sharing, adaptation, distribution and reproduction in any medium or format, as long as you give appropriate credit to the original author(s) and the source, provide a link to the Creative Commons licence, and indicate if changes were made. The images or other third party material in this article are included in the article's Creative Commons licence, unless indicated otherwise in a credit line to the material. If material is not included in the article's Creative Commons licence and your intended use is not permitted by statutory regulation or exceeds the permitted use, you will need to obtain permission directly from the copyright holder. To view a copy of this licence, visit http://creativecommons.org/licenses/by/4.0/. The Creative Commons Public Domain Dedication waiver (http://creativecommons.org/publicdomain/zero/1.0/) applies to the data made available in this article, unless otherwise stated in a credit line to the data. 


\section{Background}

Marek's disease (MD) is a lymphoproliferative disease of chickens caused by a highly oncogenic Gallid alphaherpesvirus 2, a naturally occurring alphaherpesvirus [1], which goes through a complex life cycle of four main phases [2, 3]: an early cytolytic phase at 2-7 days post infection (dpi), a latent phase around 7-10 dpi, a late cytolytic phase and finally, a proliferation phase after 28 dpi. MD currently remains a neoplastic disease of chickens of serious concerns to the global poultry industry. The control of MD mainly relies on vaccination; however, the vaccination efficacy is being reduced due to newly emerging strains of Marek's disease virus (MDV) with escalated virulence. Enhancing genetic resistance to MD in poultry is an important long-term goal to better control MD. Therefore, understanding of genetic basis of resistance to $\mathrm{MD}$ and improving $\mathrm{MD}$ resistance in chickens are of great interest for the poultry industry and animal welfare. In order to optimally implement this control strategy through marker assisted selection (MAS) and to understand the etiology and mechanisms of $\mathrm{MD}$, it is necessary to identify more specific genes and variants with respect to MD resistance.

Genetic variations play crucial roles in phenotypic diversity [4], some of which may underlie major mechanisms that account for variations in disease resistance. The identification of structural variations and potential genetic markers is very important for better understanding of disease resistance, as well as genomic prediction and genetic improvement by selection. There are several types of genetic variations, where copy number variation $(\mathrm{CNV})$ is one of the important types. According to current knowledge, $\mathrm{CNV}$ is a type of important genomic structural variation which includes intermediately sized DNA segments that have undergone submicroscopic insertion, deletion, segmental duplication, and complex changes of greater than 1 kilobases $(\mathrm{Kb})$ to several megabases $(\mathrm{Mb})$ in size [5]. It is also a major source of genetic variation underlying phenotypic diversity [6]. Following the first two genome-wide scans for CNVs in human genome [7, 8], a large number of CNV detection studies have been performed, which revealed that CNVs are ubiquitously distributed in genome and can influence the phenotype via regulations of gene expression and gene dosage [9-11]. Besides, numerous studies in other species have also shown that CNVs contributed to phenotypic variation of complex diseases and traits [12-19], including MD in chicken [20-22]. Two major traditional platforms employed in CNV detection are based on SNP chips, one is known as comparative genomic hybridization $(\mathrm{CGH})$ array, and the other is SNP genotyping array. However, due to the limitation in resolution and sensitivity, it is difficult to exhaust small CNVs shorter than $10 \mathrm{~kb}$ in length in detection and to identify the precise boundaries of CNVs.
In recent years, a variety of $\mathrm{CNV}$ detection approaches based on next-generation sequencing (NGS) were proposed, which offer promising alternatives as they have higher effective resolution and sensitivity to discover CNVs with more types and wider size ranges. Advances in NGS have enhanced the new platform for more detailed characterization of CNVs in genomes [23-25].

The primary aim of the present study is to perform a genome-wide $\mathrm{CNV}$ analysis by next generation sequencing in the effort to identify CNVs that may confer the difference in genetic resistance to MD between genetic lines. We applied deep sequencing on samples from nine different genetic chicken lines that significantly vary in genetic resistance to $\mathrm{MD}$, including two chicken inbred lines, line $6_{3}$ and line $7_{2}$, their $\mathrm{F} 1$ reciprocal cross, and the recombinant congenic strains (RCS). RCSs were developed using line $6_{3}$ as the progenitor background line and line $7_{2}$ as the progenitor donor line. Eventually, a series of 19 RCSs were generated and each contains a random sample of $87.5 \%$ line $6_{3}$ and $12.5 \%$ line $7_{2}$ genome. All of the chicken lines shared a common major histocompatibility complex $B * 2$ haplotype, but the MD resistance/susceptibility differs among the RCS [1]. Furthermore, CNVnator [26] was employed to generate a comprehensive map of CNVRs and genes. qPCR was used to validate the detected CNVRs. Our finding provides valuable insights for understanding the genetic mechanism of resistance to MD, and the identified genes and pathways could greatly facilitate further functional characterization studies.

\section{Results}

\section{Mapping statistics and CNV detection}

In this study, we performed whole genome sequencing in nine different chicken lines (Fig. 1) using Illumina paired-end libraries of 26 female chickens. The average numbers of raw reads were approximately 242.26, 230.56, 127.06, 31.39, 34.50, 42.12, 61.06, 57.68 and 37.72 million for lines $6_{3}, 7_{2}, F_{1}$, and RCS-A, D, J, L, M, $\mathrm{X}$, respectively (Additional file 1: Table S1). After quality control, an average of 30.42 to 226.30 million reads of each chicken line were successfully aligned to the reference genome (galGal4) with the mapping levels ranging from 90.04 to $98.10 \%$ for all the individuals. The sequencing effective depth varied from an average of $5.95 \times$ for six RCSs to an average of $19.84 \times$ for lines $6_{3}, 7_{2}$ and their $\mathrm{F}_{1}$ hybrid, and the average coverage with respect to the reference genome was $88.25 \%$. These high quality alignments provide confident for the subsequent analysis with a minimum of false positives.

We then applied the CNVnator software for CNV detection and the average number of CNVs per line, that passed our stringent filtering criteria, was 1888, ranging from 1368 in line RCS-A to 2476 in line RCS-J. The size 


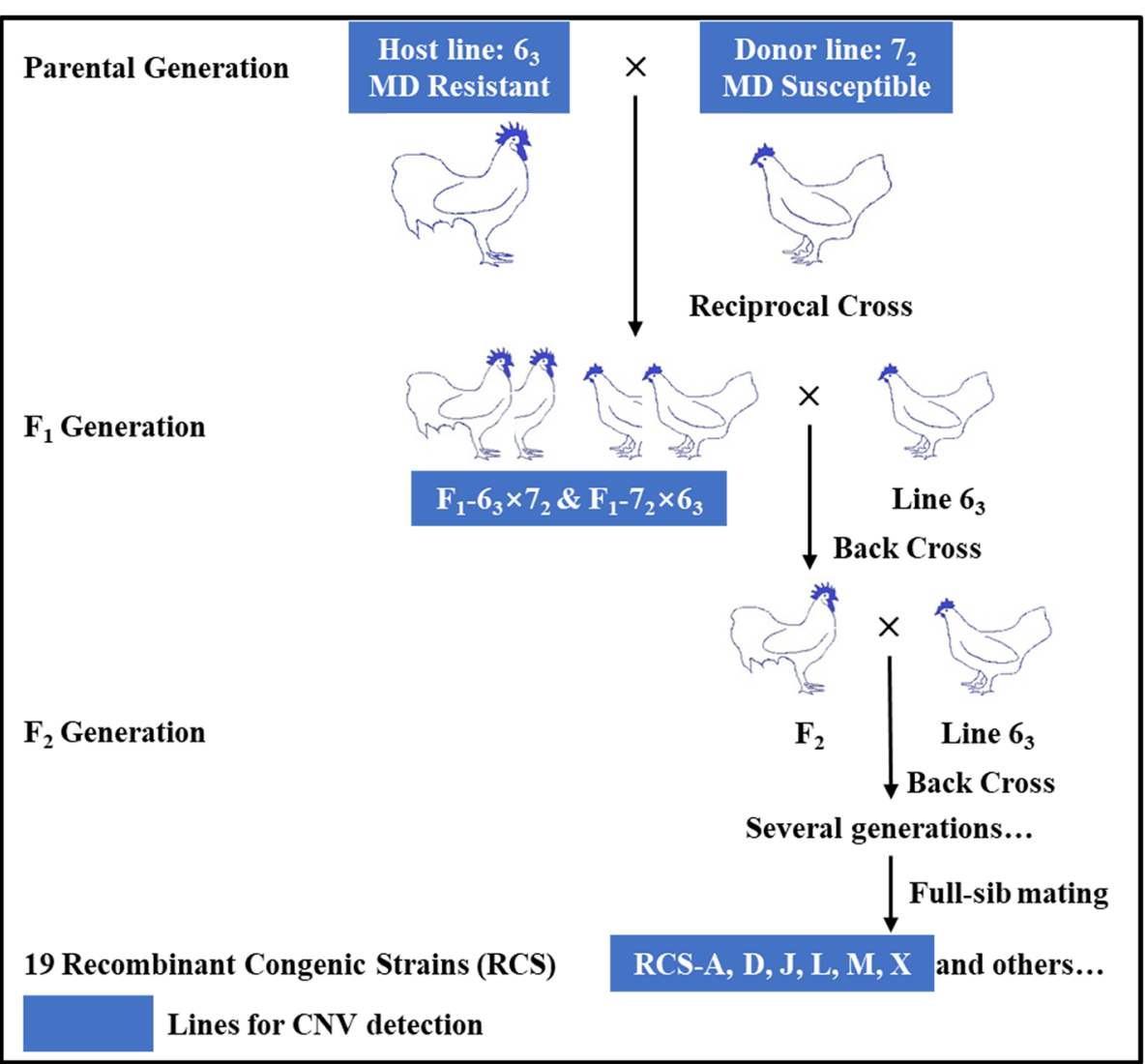

Fig. 1 The chicken population used in this study. Chicken lines labeled blue were selected for CNV detection

of these CNVs ranges from $1 \mathrm{~Kb}$ to $9.56 \mathrm{Mb}$, with an average of $95.56 \mathrm{~Kb}$. Detailed statistics of $\mathrm{CNV}$ calls are shown in Additional file 2: Table S2. A total of 1649 CNV regions (CNVRs) (Table 1) allowing for CNV overlaps of $1 \mathrm{bp}$ or greater were obtained across all the chicken lines after merging, covering autosomes 1-28, and sex chromosomes Z and W. The chicken CNV map across the genome is shown in Fig. 2. The length of CNVRs ranged from $1 \mathrm{~Kb}$ to $18.19 \mathrm{Mb}$ with an average of $0.36 \mathrm{Mb}$. In total, 1200 (72.8\%) out of all CNVRs had sizes varying from 1 to $200 \mathrm{~Kb}$ (Fig. 3a). The count of CNVRs on each chromosome was directly proportional to the chromosome length, and five macrochromosomes (chr1-5) possessed a large proportion $(874,53.0 \%)$ of all putative CNVRs. The number of CNVRs in different chicken lines varied greatly, ranging from 536 in RCS-L to 852 in RCS-A. Among all CNVRs, 495 (30.02\%) were present in only one chicken line and 90 (5.46\%) CNVRs are shared in all the nine chicken lines (Fig. 3b). In addition, the CNVRs belonging to gain and loss account

Table 1 Summary statistics of line-specific and shared CNVRs

\begin{tabular}{|c|c|c|c|c|c|c|c|c|c|c|}
\hline Group & No. of CNVRs & Gain & Loss & Total & Common & Harbored genes & Line-specific & Gain & Loss & Harbored genes \\
\hline $6_{3}$ & 1193 & 210 & 983 & 1649 & 90 & 1360 & 55 & 4 & 51 & 62 \\
\hline 72 & 1134 & 205 & 929 & & & & 44 & 7 & 37 & 57 \\
\hline$F_{1}$ & 1111 & 212 & 899 & & & & 82 & 4 & 78 & 135 \\
\hline RCS_A & 722 & 140 & 582 & & & & 15 & 1 & 14 & 41 \\
\hline RCS_D & 801 & 555 & 246 & & & & 14 & 7 & 7 & 33 \\
\hline RCS_J & 949 & 601 & 348 & & & & 72 & 23 & 49 & 300 \\
\hline RCS_L & 1031 & 812 & 219 & & & & 190 & 185 & 5 & 559 \\
\hline RCS_M & 765 & 133 & 632 & & & & 18 & 1 & 17 & 44 \\
\hline RCS_X & 895 & 617 & 278 & & & & 5 & 2 & 3 & 15 \\
\hline
\end{tabular}




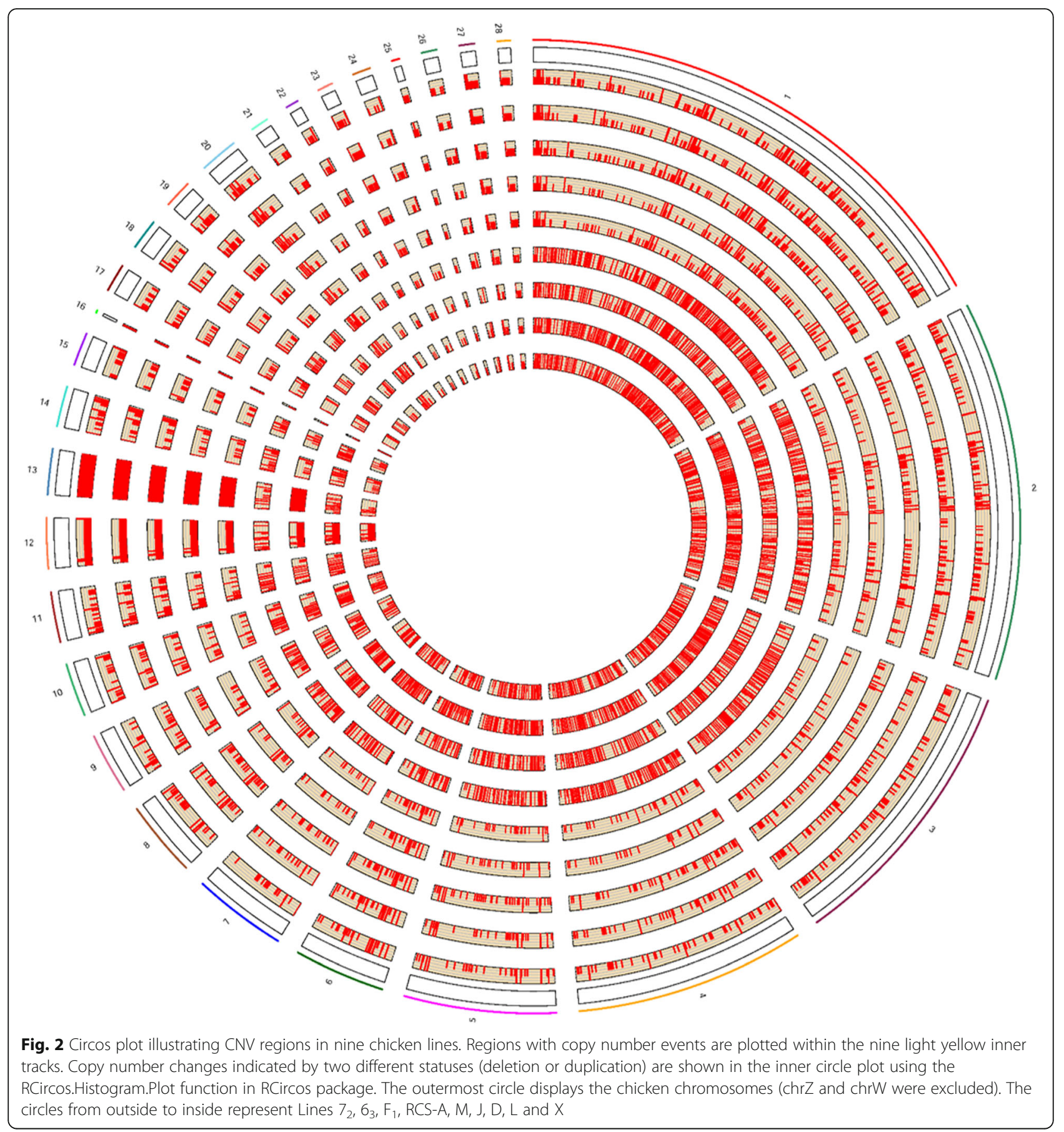

for $47.1 \%$ (776 events) and $52.9 \% \quad(873$ events), respectively.

\section{Gene annotation and functional analysis}

The genes harbored in the inferred CNVRs were extracted using custom Perl scripts. As a result, a total of 2588 RefSeq genes within the regions of the 1649 CNVRs were obtained, where a majority of these genes were involved in immunity, tumors and diseases. The identified genes were submitted to DAVID (version 6.8) for $\mathrm{GO}$ and pathway enrichment analyses. Using functional annotation clustering, at the highest classification stringency, 145 clusters were formed, where only 9 clusters were chosen after using an enrichment cutoff of $>1.0$ (Additional file 3: Table S3). GO terms and KEGG pathways analyses invoked in DAVID yielded 36 significant enriched functional terms (28 terms of biological process, 2 terms of cellular component, and 6 terms of 


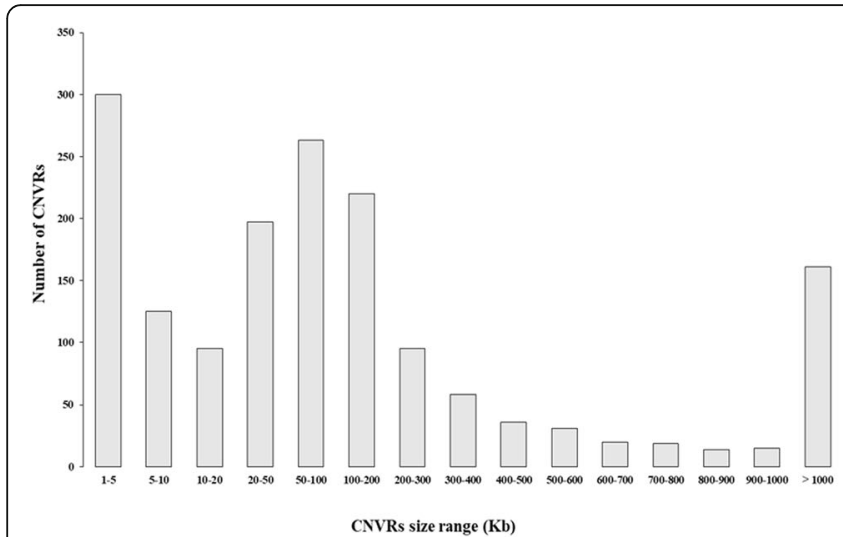

A

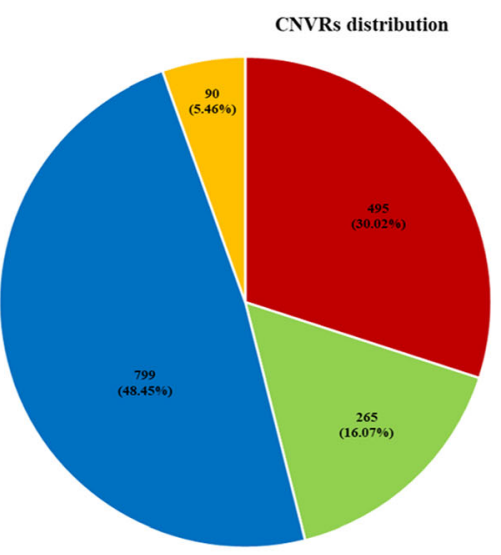

- Unique CNTRs

CNTRs shared in 2 chicken lines - CNTRs shared in 3.8 chicken lines CNVRs shared in 9 chicken lines

Fig. 3 Distribution of CNVRs in nine chicken lines. a Length distribution of CNVRs. 1200 (72.8\%) CNVR events are shorter than 200 kb. b Frequency distribution of unique and overlapped CNVRs. 495 (30.02\%) CNVRs occur in only one chicken line and 90 (5.46\%) CNVRs are shared in all the nine chicken lines

molecular function; $P<0.05$, Fig. 4). In addition, it yielded 6 significant enriched pathways $(P<0.05$, Table 2), including the JAK/STAT signaling pathway (gga04630, Additional file 4: Figure S1). The detailed information of all the GO terms and pathways are shown in Additional file 5: Table S4.

\section{PCA analysis and cluster}

To investigate genetic structure in nine inbred chicken lines, we performed a principal component analysis (PCA) using the CNV segments by custom R scripts. Nine principal components were calculated and the first two PCs were used in the plot (Fig. 5a). The nine lines

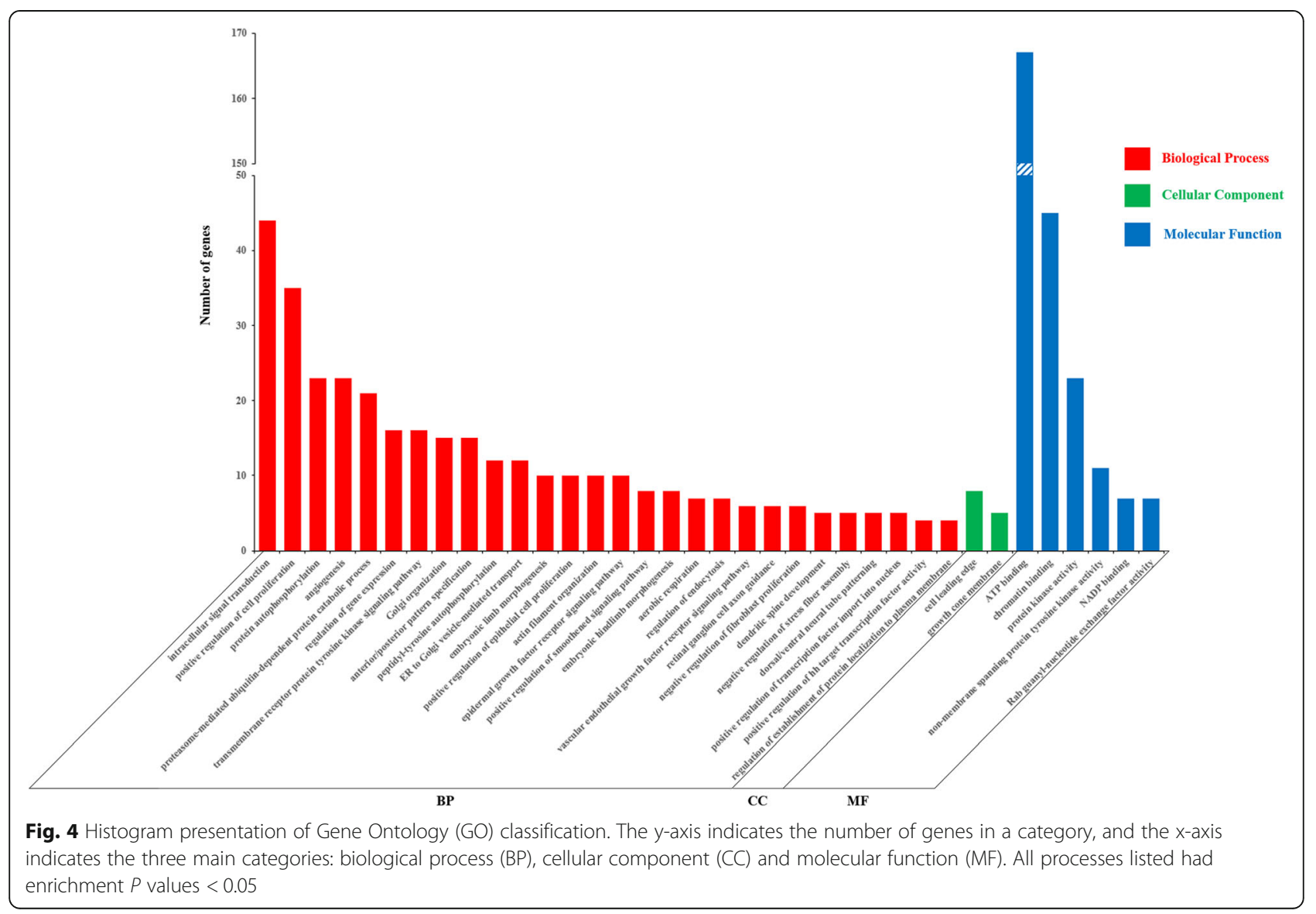


Table 2 Enriched KEGG pathways of the genes harbored in the CNVRs $(P<0.05)$

\begin{tabular}{lllll}
\hline Category & Term & Count & $\%$ & P-Value \\
\hline KEGG_PATHWAY & gga00900:Terpenoid backbone biosynthesis & 10 & 0.45 & $2.31 \mathrm{E}-03$ \\
KEGG_PATHWAY & gga04144:Endocytosis & 49 & 2.22 & $1.92 \mathrm{E}-02$ \\
KEGG_PATHWAY & gga04146:Peroxisome & 20 & 0.90 & $2.10 \mathrm{E}-02$ \\
KEGG_PATHWAY & gga04120:Ubiquitin mediated proteolysis & 28 & 1.27 & $3.06 \mathrm{E}-02$ \\
KEGG_PATHWAY & gga04630:JAK/STAT signaling pathway & 25 & 1.13 & $4.65 \mathrm{E}-02$ \\
KEGG_PATHWAY & gga04520:Adherens junction & 17 & 0.77 & $4.85 \mathrm{E}-02$ \\
\hline
\end{tabular}

were clustered to four approximate groups with the similar patterns, as indicated by dashed circles (Fig. 5a), which were consistent with their susceptibility to MD (Fig. 5b [27]). Lines RCS-A, $M$ and $7_{2}$ were well clustered together with high MD susceptibility. Lines RCS-D, J, L and X were clustered together with high resistance to MD. Interestingly enough, as expected, $F_{1}$ individuals with a medium MD resistance were in an intermediary position between line $7_{2}$ and line $6_{3}$, which provided the theoretical basis to identify imprinting genes for disease resistance.

\section{Shared versus line-specific CNVRs}

To investigate how frequently CNVRs were shared or lineage-specific across different lines, we calculated the CNVR numbers among the nine inbred chicken lines (Table 1). In total, 90 CNVRs were detected across all the individuals, which represented the commonly shared CNVRs. A total of $55,44,82,15,14,72,190,18$, and 5 CNVRs were detected as line-specific CNVRs in line $6_{3}, 7_{2}$, $\mathrm{F}_{1}$, RCS-A, D, J, L, M, and X, respectively, as compared to other lines (Table 1, Additional file 6: Table S5).
Importantly, the line $6_{3}$ and $7_{2}$ lineage-specific CNVRs could potentially offer certain clues to explore the genetic mechanisms of MD resistance or susceptibility. So, a total of 62 and 57 harbored genes were identified in line $6_{3}$ and $7_{2}$, respectively, including several immune-, tumor- and disease-related genes, such as interferon regulatory factor 2 (IRF2), suggesting that the CNV in the IRF2 gene is specific to line $7_{2}$ in this study (Additional file 7: Table S6). Interestingly, our lab also found a MD-resistance associated differentially methylated region (DMR, chr4: 38,999,001$39,000,000$ ), which was hypermethylated in line $6_{3}$ compared with line $7_{2}$, in our previous DNA methylation study. The harbored region also included IRF2, which is involved in immune response IFN alpha/beta signaling pathway. This gene could be a candidate gene associated with MD susceptibility.

\section{CNVRs validation}

To confirm the identified CNVRs, 10 CNVRs containing gains (duplications) and losses (deletions) detected here were validated by qPCR using two reference genes

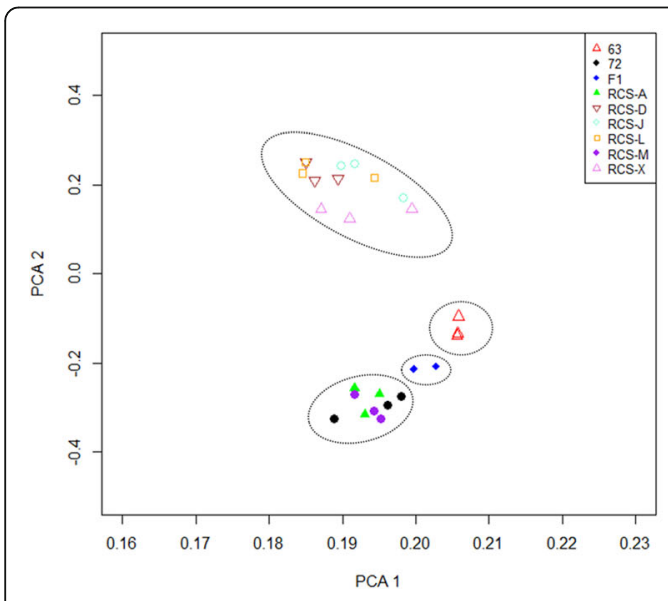

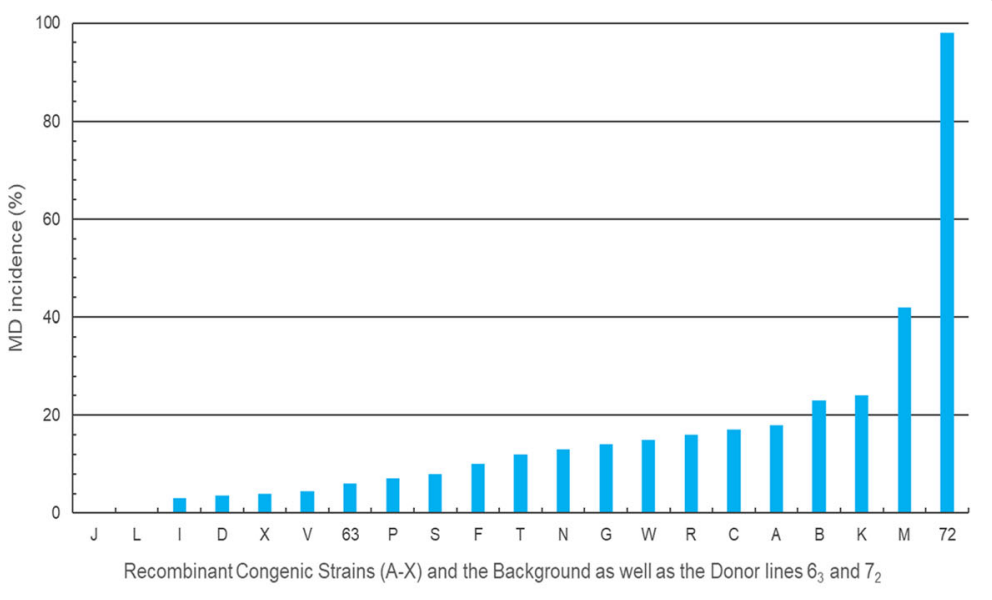

B

Fig. 5 a PCA plot based on the first two principal components in all the nine chicken lines. These nine lines were clustered to four approximate groups, as indicated by dashed circles, which were consistent with their susceptibility to MD. b Histogram plot of MD incidence (\%) rate. MD resistance in chickens is generally evaluated with MD incidence (induced gross tumors by MDV) and survival days post MDV challenge. The calculation was done as the ratio of number of birds with tumors/total number of birds challenged within each of the lines multiplied by 100 (cited from Xie et al. [27]) 
(THRSP and $\beta$-actin). We found that all of the 10 CNVRs were confirmed in agreement with the CNVnator results (Fig. 6a), further supporting the reliability of the detected CNVRs. We also performed a qPCR validation on two line $7_{2}$ lineage-specific deletion CNVRs: CNVR6 (chr4: 38,999, 001-39,000,200, harbored gene: IRF2) and CNVR7 (chr4: 82,407,001-82,409,800, harbored gene: MAX dimerization protein 4, MXD4). For CNVR6, a total deletion was detected in line $7_{2}$, while line $6_{3}$ had a normal status. For CNVR7, line $7_{2}$ had two third of the normal copy numbers, while line $6_{3}$ also had a normal status (Fig. 6b). Therefore, the copy numbers of these two loci were found significantly lower in line $7_{2}$ as compared to line $6_{3}$, again supporting our $\mathrm{CNV}$ calls and suggesting that they are potentially linked to MD susceptibility.

\section{Comparison with other studies on CNV in chickens}

Considering that most of the previous $\mathrm{CNV}$ detection studies were based on the galGal2 and galGal3 genome assembly, coordinates of the CNVRs were converted using the UCSC liftOver tool (http://genome.ucsc.edu/ cgi-bin/hgLiftOver). We migrated all chromosomal CNVRs from galGal2 and galGal3 (used in previous studies) to galGal4. We eventually obtained 585 CNVRs in the present study for comparison. Our results were then compared to 12 previous reports on chicken genomic CNV (Table 3). As a result, about 3.7, 5.2 and $4.1 \%$ of the Crooijmans et al.'s [34], Tian et al.'s [35] and Yi et al.'s [25] results can be validated in our study, respectively. Moreover, about $12.1,4.0$ and $8.8 \%$ of the Luo et al.'s [20], Yan et al.'s [21] and Xu et al.'s [22] results that also involved in MD were validated in our study. Taken together, $42.2 \%$ of our CNVRs overlapped with these three MD studies. The detailed information of CNVRs identified in this study and previous studies is provided in Additional file 8: Table S7.

\section{Discussion}

$\mathrm{MD}$, a complicated tumor disease, has been used as a model for human tumor study [36]. The genetic mechanism underlying MD is likely to be very complex and remains incompletely understood. Thus, it is important to understand the genetic basis of MD-resistance or MD-susceptibility poultry, which can provide crucial clues for human diseases. In the present study, based on the high throughput sequencing platform, some bioinformatics analyses were conducted to identify CNVs, genes and enriched pathways, taking full advantage of identical genetic background in nine inbred chicken lines.

Copy number variations in the chicken genome have been explored by many research groups in the past decade. However, most of the previous studies focused on CNV discoveries using low-density SNP arrays [30, 31]. With the development of high-throughput genotyping technology, the NGS data have been used to detect the complex diseases and traits-related $\mathrm{CNVs}$. $\mathrm{CNV}$ detections based on NGS data, which has much higher density compared to SNP chip data, have been developed and implemented in different tools [37]. There are four main methods for detecting CNVs with NGS data: Read-Pair (RP), Split Read (SR), Read Depth (RD), and assembly (AS) based methods, including CNVnator [26] used here, Pindel [38], ReadDepth [39], PEMer [40], and some other useful methods. However, each of the methods have different advantages and limitations in their applicability and suitability for NGS data. CNVnator based on RD method was the only software employed in this study. It uses the established mean-shift approach with additional corrections for multiple-bandwidth partitioning and GC correction, for
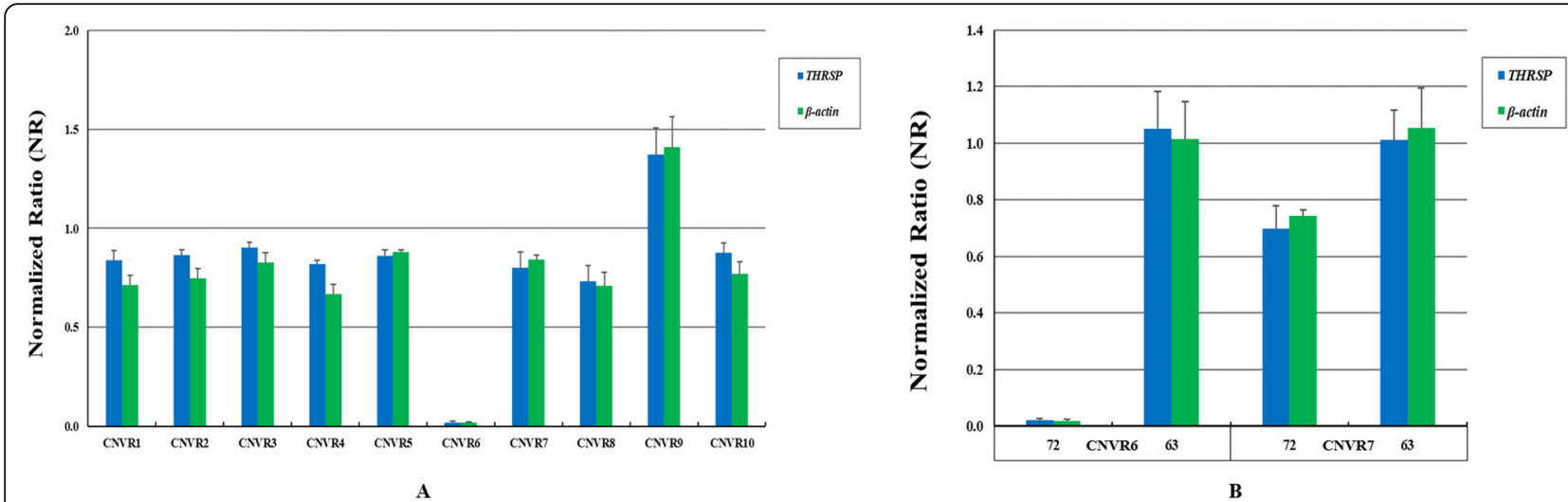

Fig. 6 qPCR validation. a Normalized ratio (NR) obtained by qPCR for 10 CNVRs. The $y$-axis shows the NR values, and the x-axis shows the CNVR ID. $\mathbf{b}$ qPCR validation on two line $7_{2}$ lineage-specific deletion CNVRs. The $y$-axis shows the NR values, and the $x$-axis shows the CNVR ID and chicken lines. THRSP and $\beta$-actin served as reference genes with no variation. Samples with NR value of approximately 1 denote normal status, samples with NR value of less than 1 denote copy-number-loss status, and samples with value of about 1.5 or more denote copy-number-gain status 
Table 3 A summary of the chicken CNVRs identified in this study and previous studies

\begin{tabular}{|c|c|c|c|c|}
\hline Study & Platform & Number of CNVRs & Total length (Mb) & $\begin{array}{l}\text { Concordant number } \\
\text { with our study }\end{array}$ \\
\hline Griffin et al., 2008 [28] & NimbleGen $385 \mathrm{~K}$ & 20 & 2.84 & 1 \\
\hline Skinner et al., 2009 [29] & NimbleGen $385 \mathrm{~K}$ & 15 & 2.91 & 2 \\
\hline Wang et al., 2010 [30] & NimbleGen $385 \mathrm{~K}$ & 96 & 16.14 & 4 \\
\hline Volker et al., 2010 [31] & NimbleGen $385 \mathrm{~K}$ & 25 & 5.29 & 1 \\
\hline Wang et al., 2012 [32, 33] & Agilent $400 \mathrm{~K}$ & 130 & 3.34 & 4 \\
\hline Crooijmans et al., 2013 [34] & Aglilent $244 \mathrm{~K}$ & 1553 & 61.67 & 57 \\
\hline Luo et al., 2013 [20] & Agilent $400 \mathrm{~K}$ & 33 & 1.92 & 4 \\
\hline Tian et al., 2013 [35] & Agilent $400 \mathrm{~K}$ & 308 & 10.81 & 16 \\
\hline Zhou et al., 2014 [15] & Illumina $60 \mathrm{~K}$ & 137 & 27.32 & 7 \\
\hline Yi et al., 2014 [25] & Illumina HiSeq 2000 & 7530 & 88.12 & 306 \\
\hline Yan et al., 2015 [21] & Illumina HiSeq 2000 & 5680 & 28.94 & 228 \\
\hline Xu et al., 2017 [22] & Affymetix $600 \mathrm{~K}$ & 170 & 0.83 & 15 \\
\hline This study & Illumina HiSeq 2000 & 585 & 88.16 & - \\
\hline
\end{tabular}

more accurate $\mathrm{CNV}$ detection. Previous approaches using $\mathrm{RD}$ were limited to only unique regions of the genome and discovered only large CNVs with poor breakpoint resolution, or could not perform genotyping. CNVnator is able to discover CNVs in a vast range of sizes, from a few hundred bp to several $\mathrm{Mb}$ in length, in the whole genome. Therefore, our results here could reveal additional novel genetic variations underlying $\mathrm{MD}$ than those revealed by SNP arrays alone.

In the present study, we performed comparisons with the previous CNV studies, especially three researches also involved in MD. We found 247 CNVRs covering 93.9 $\mathrm{Mb}$ in length that overlapped with these three MD studies. It is interesting to note that only $7(1.2 \%)$ and 15 (2.6\%) CNVRs were shared with Luo et al.'s [20] and $\mathrm{Xu}$ et al.'s [22] results using SNP chip data, which may be, in part, related to limited sample sizes, different platforms, different analysis methods, and different chicken genome references (although we converted the genome positions from two previous genome assemblies (galGal2 and galGal3) to a newer one (galGal4) with the help of LiftOver based on UCSC, some information may still be missing). More importantly, we also used different chicken lines, especially the selected RCSs from a total of 19 RCSs (Fig. 1). We also compared with the CNV identified in lines $6_{3}$ and $7_{2}$ using NGS data for MD study [21], and found that 228 (39.0\%) CNVRs overlapped, which provided more effective information for our study. Moreover, our study explored the genetic structure based on CNV in different inbred chicken lines. The PCA showed clearly that the first two PCs can divide all chickens into four unique groups, which is similar to the results of $\mathrm{Xu}$ et al. [22]. Therefore, our study further confirms that CNV markers can be used to study the genetic variability in diverse chicken lines, which could possibly contribute to lineage-specific phenotypes.

The genetic mechanism underlying MD is likely to be very complex and is not clear yet. It may be determined by some specific structural variations but not a single gene or a SNP mutation, though several candidate genes have been reported in previous studies described above. In the current study, we investigated CNVs among diverse inbred lines and found 55 and 44 unique CNVRs in lines $6_{3}$ and $7_{2}$, respectively, which could be associated with MD. Notably, we successfully identified a CNVR, which was a deletion and a normal copy number in all individuals from line $7_{2}$ and line $6_{3}$, respectively, including a nearby gene IRF2. Fortunately, the IRF2 gene was also highlighted in our previous DNA methylation study involved in a critical DMR identified by methylCpG binding domain protein enriched genome sequencing (MBD-seq) with a false discovery rate $(\mathrm{FDR})<0.1$ and validated by bisulfite cloning sequencing, which was hypermethylated in line $6_{3}$ compared with line $7_{2}$. The region of the DMR identified in previous study and the CNVR identified here was almost completely the same, with the same start site and a $1000 \mathrm{bp}$ overlaps. The nearby gene IRF2 is a disease- and virus-related gene involved in the interferon gamma signaling pathway and the immune response IFN alpha/beta signaling pathway [41]. This gene is conserved in human and some other species like chimpanzee, Rhesus monkey, dog, cow, mouse, rat, zebrafish, and frog. Thus, some mutations or structural variations of this gene could be key factors related to disorders or diseases. It was reported that IRF2 gene was associated with several diseases in chickens like necrotic enteritis [42], pancreatic cancer [43], and atopic dermatitis and eczema herpeticum [44]. More interestingly, this gene can specifically bind to the upstream 
regulatory region of type I IFN and IFN-inducible MHC class I genes, which could be an important clue to explore the genetic mechanisms of MD resistance because, to our knowledge, MHC plays an important role in the determination of resistance to MD [1]. Therefore, IRF2 may be a very important gene related to $\mathrm{MD}$ according to the structural variation identified here, its known functions and our former studies. Another useful information obtained in this study is the JAK/STAT signaling pathway, which was also considered as a potential pathway responding to MDV infection reported by Perumbakkam et al. [45]. The JAK/STAT signaling pathway is one of a handful of pleiotropic cascades used to transduce a multitude of signals for development and homeostasis in animals [46]. JAK activation stimulates cell proliferation, differentiation, cell migration and apoptosis. These cellular events are critical to immune development and some other processes. Importantly, mutations that constitutively activate or fail to regulate JAK signaling properly cause inflammatory disease, including several chicken diseases [47, 48]. Additionally, a previous study reported that IRF2 can regulate macrophage apoptosis through a STAT1/3 [49], which provides valuable and potential interaction of IRF2 and JAK/STAT pathway and might jointly contribute to the genetic resistance to MD. Therefore, we hypothesize a probable mechanism of complex disease: the deletions in $\mathrm{CNV}$ could be associated with different epigenetic effects, which further regulate an interacting pathway leading to occurrence of diseases.

\section{Conclusions}

In summary, we investigated copy number variations in inbred chicken lines using next generation sequencing. We have successfully identified a number of line-specific CNVRs, as well as revealed genes and pathways that may be involved in genetic resistance to MD. Combined with our previous study and due to the complexity of MD, we ultimately found a high-confidence candidate gene IRF2, and an immune- or disease-related pathway, JAK/STAT signaling pathway, which could jointly play potentially important roles in response to MD resistance. Overall, our findings in the present study will provide valuable insights for understanding the genetic mechanism of resistance to MD and will be worthy of further functional characterization.

\section{Methods}

\section{Experimental population}

A total of 26 female chickens without treatments were used for blood collection in this study, including three chickens from each of the line $6_{3}$ (MD-resistant), line $7_{2}$ (MD-susceptible) and six recombinant congenic strains (RCSs, RCS-A, D, J, L, M, and X), and two chickens from reciprocal cross $F_{1}$ hybrid $6_{3} \times 7_{2}$ (USDA-ARS ADOL, East Lansing, Michigan, USA) [50]. RCSs were developed using line $6_{3}$ as the parental strain mated to line $7_{2}$ and then backcrossed to line $6_{3}$ twice followed by full-sib mating for more than 20 generations (Fig. 1). Eventually, diverse RCSs were generated and they contain $87.5 \%$ of line $6_{3}$ and $12.5 \%$ of line $7_{2}$ in the genetic background but differ in MD resistance/susceptibility [51]. All the experimental chickens were anesthetized with sodium pentobarbital (intraperitoneal injection: $150 \mathrm{mg} / \mathrm{kg}$ ) at the end of this study.

\section{Library construction and sequencing}

Blood samples were collected from the brachial vein by venipuncture. Genomic DNA (gDNA) from blood samples was extracted using the DNeasy Blood \& Tissue Mini Kit (Qiagen, USA) according to the manufacturer's instructions. The purity and concentration of the gDNA samples were measured by NanoDrop ND-1000 spectrophotometer (Thermo Scientific, USA) and by agarose gel electrophoresis. After the examinations, paired-end libraries were generated for each eligible sample using standard procedures (Illumina, USA). The average insert size was $500 \mathrm{bp}$, and the average read length was $100 \mathrm{bp}$ for line $6_{3}$ and line $7_{2}$, and $150 \mathrm{bp}$ for the remaining chicken lines. All libraries were sequenced on an Illumina $^{\circ}$ HiSeq 2000 sequencing platform to an average raw read sequence coverage of $\times 20$ for lines $6_{3}, 7_{2}$ and their $F_{1}$ hybrid, and $\times 6$ for the six RCSs, respectively. The depth ensured the accuracy and reduced the falsenegative rate of $\mathrm{CNV}$ calling for downstream analysis. Library preparation and all Illumina runs were performed as the standard manufacturer's protocols.

\section{Read alignment and CNV calling}

Chicken genome assembly (galGal4) was retrieved from the UCSC Genome Browser website (http://hgdownload. soe.ucsc.edu/goldenPath/galGal4/bigZips/) [52]. In order to minimize the mapping errors, quality control was performed by FastQC [53] and low quality reads were removed with the help of FastX Toolkit (Gordon A and Hannon G: Fastx-toolkit: FASTQ/A short-reads preprocessing tools, unpublished) and Trimmomatic using the default parameters. The resulting FastQ files of mapping reads of each sample were aligned to the reference genome individually using Burrows-Wheeler Aligner (BWAMEM) (v0.7.15) [54] with mainly default parameters. SAMtools (v1.3) [55] was then used to convert the alignment results (SAM format) to BAM format and all converted BAM files were sorted with the command SAMtools. Duplicate reads were removed from individual sample alignments using MarkDuplicates in the Picard package (http://broadinstitute.github.io/picard/) to avoid any influence on variant detection, and reads were merged using MergeSam-Files. We additionally performed local realignment using Genome Analysis 
Toolkit (GATK, v3.5) [56] to enhance the alignments in regions of indel polymorphisms, which can greatly improve the sensitivity and specificity in CNV calling [57].

After mapping, CNV calling was performed using CNVnator (v0.3.3) software [26] based on read depth (RD) method to predict genomic CNVs between the nine chicken lines and the reference. CNVnator firstly calculated the counts of mapped reads within user specified non-overlapping bins of equal size as the RD signal, and then adjusted the signal in consideration of the potential correlation between RD signal and GC content of the underlying genomic sequence. The mean-shift algorithm was employed to segment the signal with presumably different underlying $\mathrm{CN}$. Then $\mathrm{CNV}$ s were predicted by applying statistical significance tests to the segments. We then ran CNVnator with a bin size of 100 bp for our data. CNV calls were filtered using stringent criteria including $P$-value $<0.05$ and minimum size $>1$ $\mathrm{Kb}$, and calls with $>50 \%$ of q0 (zero mapping quality) reads within the $\mathrm{CNV}$ regions were removed (q0 filter). All CNV calls overlapping with gaps in the reference genome were excluded from consideration. CNVs located on random contigs (chrN_random), unlocalized chromosomes (chrUn), or in overlapping gaps were discarded for further analysis due to the shorter length of the chrUn contigs and mapping ambiguity of chrUn sequence reads. In order to compare our results with previous studies, we converted all chromosomal CNVRs from galGal2 and galGal3 (used in previous studies) to galGal4 with the assistance of LiftOver based on UCSC (http://genome. ucsc.edu/cgi-bin/hgLiftOver).

\section{Gene detection and functional analysis}

Results from CNVnator were combined to obtain a collective set of unique CNVs with different start or end coordinates. These CNVs were then merged into nonoverlapping CNV regions (CNVRs) by aggregating CNVs that overlap by at least $1 \mathrm{bp}$ across all samples of each chicken line. The Ensembl genes (release 85 Database) were obtained using BioMart software based on the chicken gene sequence assembly (galGal4) and the genes harbored in the inferred CNVRs were extracted using custom Perl scripts. The Database for Annotation, Visualization, and Integrated Discovery (DAVID, version 6.8) (https://david.ncifcrf.gov/) [58] was used to perform the gene ontology (GO) enrichment analysis and Kyoto Encyclopedia of Genes and Genomes (KEGG) pathway analysis.

Validation of CNVRs by quantitative real-time PCR (qPCR) To experimentally validate the detected $\mathrm{CNV}$ calls by CNVnator, we performed qPCR confirmation of ten CNVRs randomly selected from line $6_{3}$ and line $7_{2}$, respectively, using gDNA samples from different chicken lines. All the primers were designed based on the GenBank reference sequences using the Primer 3.0 webtool (http://frodo.wi. mit.edu/primer3/) (Additional file 9: Table S8). The $\beta$-actin gene and thyroid hormone responsive (THRSP) gene served as reference genes. For each chicken line, at least three individuals were used to do the validation. qPCR using SYBR Green PCR Kit was performed in triplicate based on iCycler iQ PCR System (Bio-Rad). qPCR program was run as follows: pre-incubation $\left(95^{\circ} \mathrm{C}\right.$ for $\left.10 \mathrm{~min}\right), 40$ cycles of amplification $\left(95^{\circ} \mathrm{C}\right.$ for $10 \mathrm{~s}, 60^{\circ} \mathrm{C}$ for $10 \mathrm{~s}$, and $72^{\circ} \mathrm{C}$ for $\left.10 \mathrm{~s}\right)$, melting curves using a heat ramp and cool down. Cycle threshold values (Ct values) were obtained from iCycler iQ PCR software. The $2^{-\Delta \Delta C T}$ method was used to calculate the copy number [59-61]. The corresponding equation was:

$$
\begin{aligned}
\Delta \Delta C T & =\left(C T_{\text {target gene }}-C T_{\text {reference gene }}\right)_{\text {sample } A} \\
& -\left(C T_{\text {target gene }}-C T_{\text {reference gene }}\right)_{\text {sample } B},
\end{aligned}
$$

where CT is the threshold cycle, sample A is the tested individual, and sample $\mathrm{B}$ is the control individual with single copy or no variation in copy number. Samples with Normal Ratio (NR) about 1 denote normal individuals (two copies), samples with NR of less than 1 denote one copy loss individuals, and samples with NR about 1.5 or more denote copy number gain individuals $[32,33]$.

\section{Supplementary information}

Supplementary information accompanies this paper at https://doi.org/10. 1186/s12863-020-00884-W

Additional file 1: Table S1. Summary statistics for sequencing.

Additional file 2: Table S2. Summary statistics of raw and screened CNVs.

Additional file 3: Table S3. The detailed lists of clusters with enrichment scores $>1.0$ from DAVID.

Additional file 4: Figure S1. JAK/STAT signaling pathway. Additional file 5: Table S4. The detailed lists of all the GO terms and pathways from DAVID.

Additional file 6: Table S5. Lists of line-specific and shared CNVRs in all the chicken lines.

Additional file 7: Table S6. Lists of disease-related genes in line $\sigma_{3}$ and line 72 , respectively.

Additional file 8: Table S7. The detailed information of CNVRs identified in this study and previous studies.

Additional file 9: Table S8. All the primer sequences used in $\mathrm{QPCR}$ for CNVRs validation.

\section{Abbreviations}

ADOL: Avian disease and oncology laboratory; CGH: Comparative genomic hybridization;" chr: Chromosome; CNV: Copy number variation; CNVR: Copy number variation region; DAVID: The database for annotation visualization and integrated discovery; DMR: Differentially methylated region; dpi: Days post infection; FDR: False discovery rate; gDNA: Genomic DNA; GO: Gene ontology; IRF2: Interferon regulatory factor 2; Kb: Kilobases; KEGG: Kyoto encyclopedia of genes and genomes; Mb: Megabases; MBD-seq: Methyl-CpG binding domain protein enriched genome sequencing; MD: Marek's disease; MDV: Marek's disease virus; MXD4: MAX dimerization protein 4; NGS: Next 
generation sequencing; PCA: Principal component analysis; RCS: Recombinant congenic strain; THRSP: Thyroid hormone responsive

\section{Acknowledgements}

We are deeply grateful to the Poultry Coordinators from NRSP8: National Animal Genome Research Program.

\section{Authors' contributions}

$J S, J C, H Z, N Y$ and $H B$ conceived and designed the experiments. YD, QC and $\mathrm{HZ}$ performed the experiments and participated in the data collection. $\mathrm{HB}$ and $\mathrm{YH}$ analyzed the data. $\mathrm{YH}$ and $\mathrm{YD}$ contributed reagents/materials/ analysis tools. HB wrote the paper. JS, JC, HZ, HHC, LL, EMH and HB revised the manuscript. All authors read and approved the final manuscript.

\section{Funding}

This project was supported by the National Research Initiative Competitive Grant (No. USDA-NRI/NIFA 2010-65205-20588) from the USDA National Institute of Food and Agriculture. The funding bodies played no role in the design of the study and collection, analysis, and interpretation of data and in writing the manuscript.

\section{Availability of data and materials}

The sequencing data have been submitted to the NCBI Sequence Read Archive (SRA), and are accessible through the accession number PRJNA641254.

\section{Ethics approval and consent to participate}

All of the chickens were kept in a pathogen-free facility at the Avian Disease and Oncology Laboratory (ADOL). All animals were approved by the United States Department of Agriculture, Agricultural Research Service, ADOL Animal Care and Use Committee and followed their guidelines (revised April 2005) as well as the Guide for the Care and Use of Laboratory Animals published by Institute for Laboratory Animal Research (ILAR Guide) in 1996 (http:// www.nap.edu/openbook.php?record_id=5140) and University of Maryland (R-08-62). All efforts were made to minimize discomfort and suffering.

\section{Consent for publication}

\section{Not applicable.}

\section{Competing interests}

The authors declare that they have no competing interests.

\section{Author details}

'Joint International Research Laboratory of Agriculture and Agri-Product Safety, the Ministry of Education of China, Institutes of Agricultural Science and Technology Development, Yangzhou University, Yangzhou 225009, China. ${ }^{2}$ Department of Animal \& Avian Sciences, University of Maryland, College Park, MD 20742, USA. ${ }^{3}$ Key Laboratory of Animal (Poultry) Genetics Breeding and Reproduction, Ministry of Agriculture, Institute of Animal Science, Chinese Academy of Agricultural Sciences, Beijing 100193, China. ${ }^{4}$ Department of Human Nutrition, Food and Animal Sciences, University of Hawaii at Manoa, Honolulu, HI 96822, USA. ${ }^{5}$ Institute of Animal Husbandry and Veterinary Medicine, Beijing Academy of Agriculture and Forestry Sciences, Beijing 100097, China. ${ }^{6}$ Department of Animal Genetics and Breeding, College of Animal Science and Technology, China Agricultural University, Beijing 100193, China. ${ }^{7}$ Faculty of Health Sciences, Jerusalem College of Technology, 9116001 Jerusalem, Israel. ${ }^{8}$ USDA, Agricultural Research Service, Avian Disease and Oncology Laboratory, East Lansing, MI 48823, USA.

\section{Received: 29 July 2019 Accepted: 5 July 2020}

\section{Published online: 16 July 2020}

\section{References}

1. Bacon L, Hunt $H$, Cheng $H$. A review of the development of chicken lines to resolve genes determining resistance to diseases. Poult Sci. 2000;79(8):1082-93.

2. Calnek B, Witter RL. Marek's disease-a model for herpesvirus oncology. CRC Crit Rev Microbiol. 1985;12(4):293-320.

3. Calnek B. Pathogenesis of Marek's disease virus infection. In: Marek's Disease. Berlin: Springer; 2001:25-55.
4. Weischenfeldt J, Symmons O, Spitz F, Korbel JO. Phenotypic impact of genomic structural variation: insights from and for human disease. Nat Rev Genet. 2013;14(2):125.

5. Redon $R$, Ishikawa S, Fitch KR, Feuk L, Perry GH, Andrews TD, Fiegler $H_{\text {, }}$ Shapero $\mathrm{MH}$, Carson AR, Chen W. Global variation in copy number in the human genome. Nature. 2006;444(7118):444-54.

6. Zhang F, Gu W, Hurles ME, Lupski JR. Copy number variation in human health, disease, and evolution. Annu Rev Genomics Hum Genet. 2009;10:451-81.

7. lafrate AJ, Feuk L, Rivera MN, Listewnik ML, Donahoe PK, Qi Y, Scherer SW, Lee $C$. Detection of large-scale variation in the human genome. Nat Genet. 2004;36(9):949-51.

8. Sebat J, Lakshmi B, Troge J, Alexander J, Young J, Lundin P, Månér S, Massa $\mathrm{H}$, Walker M, Chi M. Large-scale copy number polymorphism in the human genome. Science. 2004;305(5683):525-8.

9. Fiegler H, Redon R, Andrews D, Scott C, Andrews R, Carder C, Clark R, Dovey $\mathrm{O}$, Ellis $\mathrm{P}$, Feuk L. Accurate and reliable high-throughput detection of copy number variation in the human genome. Genome Res. 2006;16(12):1566-74.

10. Glessner JT, Wang K, Cai G, Korvatska O, Kim CE, Wood S, Zhang H, Estes A, Brune CW, Bradfield JP. Autism genome-wide copy number variation reveals ubiquitin and neuronal genes. Nature. 2009;459(7246):569-73.

11. Conrad DF, Pinto D, Redon R, Feuk L, Gokcumen O, Zhang Y, Aerts J, Andrews TD, Barnes C, Campbell P. Origins and functional impact of copy number variation in the human genome. Nature. 2010;464(7289):704-12.

12. Fontanesi L, Beretti F, Riggio V, Gómez González E, Dall'Olio S, Davoli R, Russo V, Portolano B. Copy number variation and missense mutations of the agouti signaling protein (ASIP) gene in goat breeds with different coat colors. Cytogenet Genome Res. 2009;126(4):333-47.

13. Wright D, Boije H, Meadows JR, Bed'Hom B, Gourichon D, Vieaud A, TixierBoichard M, Rubin C-J, Imsland F, Hallböök F. Copy number variation in intron 1 of SOX5 causes the pea-comb phenotype in chickens. PLOS Genet. 2009;5(6):e1000512.

14. Bickhart DM, Hou Y, Schroeder SG, Alkan C, Cardone MF, Matukumalli LK, Song J, Schnabel RD, Ventura M, Taylor JF. Copy number variation of individual cattle genomes using next-generation sequencing. Genome Res. 2012;22(4):778-90

15. Zhou W, Liu R, Zhang J, Zheng M, Li P, Chang G, Wen J, Zhao G. A genome-wide detection of copy number variation using SNP genotyping arrays in Beijing-you chickens. Genetica. 2014;142(5):441-50.

16. Yi G, Qu L, Chen S, Xu G, Yang N. Genome-wide copy number profiling using high-density SNP array in chickens. Anim Genet. 2015;46(2):148-57.

17. Wu Y, Fan H, Jing S, Xia J, Chen Y, Zhang L, Gao X, Li J, Gao H, Ren H. A genome-wide scan for copy number variations using high-density single nucleotide polymorphism array in Simmental cattle. Anim Genet. 2015;46(3):289-98.

18. Zhu C, Fan H, Yuan Z, Hu S, Ma X, Xuan J, Wang H, Zhang L, Wei C, Zhang Q. Genome-wide detection of CNVs in Chinese indigenous sheep with different types of tails using ovine high-density 600K SNP arrays. Sci Rep. 2016;6:27822.

19. Bai H, Sun Y, Liu N, Liu Y, Xue F, Li Y, Xu S, Ni A, Ye J, Chen Y. Genome-wide detection of CNV $s$ associated with beak deformity in chickens using highdensity 600K SNP arrays. Anim Genet. 2018;49(3):226-36.

20. Luo J, Yu Y, Mitra A, Chang S, Zhang H, Liu G, Yang N, Song J. Genome-wide copy number variant analysis in inbred chickens lines with different susceptibility to Marek's disease. G3: genes. Genomes, Genet. 2013;3(2):217-23.

21. Yan Y, Yang N, Cheng HH, Song J, Qu L. Genome-wide identification of copy number variations between two chicken lines that differ in genetic resistance to Marek's disease. BMC Genomics. 2015;16(1):843.

22. Xu L, He Y, Ding Y, Sun G, Carrillo J, Li Y, Ghaly M, Ma L, Zhang H, Liu G. Characterization of copy number variation's potential role in Marek's disease. Int J Mol Sci. 2017;18(5):1020.

23. Zhan B, Fadista J, Thomsen B, Hedegaard J, Panitz F, Bendixen C. Global assessment of genomic variation in cattle by genome resequencing and high-throughput genotyping. BMC Genomics. 2011;12(1):557.

24. Clop A, Vidal O, Amills M. Copy number variation in the genomes of domestic animals. Anim Genet. 2012;43(5):503-17.

25. Yi G, Qu L, Liu J, Yan Y, Xu G, Yang N. Genome-wide patterns of copy number variation in the diversified chicken genomes using next-generation sequencing. BMC Genomics. 2014;15(1):962.

26. Abyzov A, Urban AE, Snyder M, Gerstein M. CNVnator: an approach to discover, genotype, and characterize typical and atypical CNVs from family and population genome sequencing. Genome Res. 2011;21(6):974-84. 
27. Xie Q, Chang S, Dong K, Dunn JR, Song J, Zhang H. Genomic variation between genetic lines of white leghorns differed in resistance to marek's disease. J Clin Epigenetics. 2017;3(3):29.

28. Griffin DK, Robertson LB, Tempest HG, Vignal A, Fillon V, Crooijmans RP, Groenen MA, Deryusheva S, Gaginskaya E, Carré W. Whole genome comparative studies between chicken and turkey and their implications for avian genome evolution. BMC Genomics. 2008;9:168.

29. Skinner BM, Robertson LB, Tempest HG, Langley EJ, loannou D, Fowler KE, Crooijmans RP, Hall AD, Griffin DK, Völker M. Comparative genomics in chicken and Pekin duck using FISH mapping and microarray analysis. BMC genomics. 2009;10:357.

30. Wang X, Nahashon S, Feaster TK, Bohannon-Stewart A, Adefope N. An initial map of chromosomal segmental copy number variations in the chicken. BMC Genomics. 2010;11(1):351.

31. Völker M, Backström N, Skinner BM, Langley EJ, Bunzey SK, Ellegren H, Griffin DK. Copy number variation, chromosome rearrangement, and their association with recombination during avian evolution. Genome Res. 2010; 20(4):503-11.

32. Wang J, Jiang J, Fu W, Jiang L, Ding X, Liu J-F, Zhang Q. A genome-wide detection of copy number variations using SNP genotyping arrays in swine. BMC Genomics. 2012;13(1):273.

33. Wang Y, Gu X, Feng C, Song C, Hu X, Li N. A genome-wide survey of copy number variation regions in various chicken breeds by array comparative genomic hybridization method. Anim Genet. 2012;43(3):282-9.

34. Crooijmans RP, Fife MS, Fitzgerald TW, Strickland S, Cheng HH, Kaiser P, Redon R, Groenen MA. Large scale variation in DNA copy number in chicken breeds. BMC Genomics. 2013;14(1):398

35. Tian M, Wang Y, Gu X, Feng C, Fang S, Hu X, Li N. Copy number variants in locally raised Chinese chicken genomes determined using array comparative genomic hybridization. BMC Genomics. 2013;14(1):262

36. Osterrieder N, Kamil JP, Schumacher D, Tischer BK, Trapp S. Marek's disease virus: from miasma to model. Nat Rev Microbiol. 2006:4(4):283-94.

37. Pirooznia M, Goes FS, Zandi PP. Whole-genome CNV analysis: advances in computational approaches. Front Genet. 2015;6:138.

38. Ye K, Schulz MH, Long Q, Apweiler R, Ning Z. Pindel: a pattern growth approach to detect break points of large deletions and medium sized insertions from paired-end short reads. Bioinformatics. 2009;25(21):2865-71.

39. Miller CA, Hampton O, Coarfa C, Milosavljevic A. ReadDepth: a parallel R package for detecting copy number alterations from short sequencing reads. PLoS One. 2011;6(1):e16327.

40. Korbel JO, Abyzov A, Mu XJ, Carriero N, Cayting P, Zhang Z, Snyder M, Gerstein MB. PEMer: a computational framework with simulation-based error models for inferring genomic structural variants from massive pairedend sequencing data. Genome Biol. 2009;10(2):R23.

41. Mizutani T, Tsuji K, Ebihara Y, Taki S, Ohba Y, Taniguchi T, Honda K. Homeostatic erythropoiesis by the transcription factor IRF2 through attenuation of type I interferon signaling. Exp Hematol. 2008;36(3):255-64.

42. Broom $\sqcup$, Kogut $\mathrm{MH}$. Deciphering desirable immune responses from disease models with resistant and susceptible chickens. Poult Sci. 2018;98(4):1634-42.

43. Cui L, Deng Y, Rong Y, Lou W, Mao Z, Feng Y, Xie D, Jin D. IRF-2 is overexpressed in pancreatic cancer and promotes the growth of pancreatic cancer cells. Tumor Biol. 2012;33(1):247-55.

44. Gao P-S, Leung DY, Rafaels NM, Boguniewicz M, Hand T, Gao L, Hata TR, Schneider LC, Hanifin JM, Beaty TH. Genetic variants in interferon regulatory factor 2 (IRF2) are associated with atopic dermatitis and eczema herpeticum. J Investig Dermatol. 2012;132(3):650-7.

45. Perumbakkam S, Muir WM, Black-Pyrkosz A, Okimoto R, Cheng HH Comparison and contrast of genes and biological pathways responding to Marek's disease virus infection using allele-specific expression and differential expression in broiler and layer chickens. BMC Genomics. 2013;14(1):64

46. Rawlings JS, Rosler KM, Harrison DA. The JAK/STAT signaling pathway. J Cell Sci. 2004;117(8):1281-3.

47. Sandford EE, Orr M, Balfanz E, Bowerman N, Li X, Zhou H, Johnson TJ, Kariyawasam S, Liu P, Nolan LK. Spleen transcriptome response to infection with avian pathogenic Escherichia coli in broiler chickens. BMC Genomics. 2011:12(1):469.

48. Lin J, Xia J, Zhang K, Yang Q. Genome-wide profiling of chicken dendritic cell response to infectious bursal disease. BMC Genomics. 2016;17(1):878.

49. Cuesta N, Nhu QM, Zudaire E, Polumuri S, Cuttitta F, Vogel SN. IFN regulatory factor-2 regulates macrophage apoptosis through a STAT1/3-and caspase-1-dependent mechanism. J Immunol. 2007;178(6):3602-11.
50. Stone HA. Use of highly inbred chickens in research. Washington DC: USDA Agriculture Research Service Technical Bulletin; 1975.

51. Chang S, Ding Z, Dunn JR, Lee LF, Heidari M, Song J, Ernst CW, Zhang H. A comparative evaluation of the protective efficacy of $\mathrm{rMd} 5 \Delta \mathrm{Meq}$ and CVI988/Rispens against a vv+ strain of Marek's disease virus infection in a series of recombinant congenic strains of white Leghorn chickens. Avian Dis. 2011;55(3):384-90.

52. Meyer LR, Zweig AS, Hinrichs AS, Karolchik D, Kuhn RM, Wong M, Sloan CA, Rosenbloom KR, Roe G, Rhead B. The UCSC genome browser database: extensions and updates 2013. Nucleic Acids Res. 2013;41(D1):D64-9.

53. Schmieder R, Edwards R. Quality control and preprocessing of metagenomic datasets. Bioinformatics. 2011;27(6):863-4.

54. Bolger AM, Lohse M, Usadel B. Trimmomatic: a flexible trimmer for Illumina sequence data. Bioinformatics 2014:30(15):2114-2120.

55. Li H, Handsaker B, Wysoker A, Fennell T, Ruan J, Homer N, Marth G, Abecasis $\mathrm{G}$, Durbin R. The sequence alignment/map format and SAMtools. Bioinformatics. 2009;25(16):2078-9.

56. McKenna A, Hanna M, Banks E, Sivachenko A, Cibulskis K, Kernytsky A, Garimella K, Altshuler D, Gabriel S, Daly M. The genome analysis toolkit: a MapReduce framework for analyzing next-generation DNA sequencing data. Genome Res. 2010;20(9):1297-303.

57. DePristo MA, Banks E, Poplin R, Garimella KV, Maguire JR, Hartl C, Philippakis AA, Del Angel G, Rivas MA, Hanna M. A framework for variation discovery and genotyping using next-generation DNA sequencing data. Nat Genet. 2011;43(5):491.

58. Huang DW, Sherman BT, Lempicki RA. Systematic and integrative analysis of large gene lists using DAVID bioinformatics resources. Nat Protoc. 2009;4(1):44-57.

59. Livak KJ, Schmittgen TD. Analysis of relative gene expression data using real-time quantitative $P C R$ and the $2-\Delta \Delta C T$ method. Methods. 2001; 25(4):402-8.

60. Bodin L, Beaune PH, Loriot M-A. Determination of cytochrome P450 2D6 (CYP2D6) gene copy number by real-time quantitative PCR. Biomed Res Int. 2005;2005(3):248-53.

61. D'haene B, Vandesompele J, Hellemans J. Accurate and objective copy number profiling using real-time quantitative PCR. Methods. 2010;50(4):262-70.

\section{Publisher's Note}

Springer Nature remains neutral with regard to jurisdictional claims in published maps and institutional affiliations.

Ready to submit your research? Choose BMC and benefit from:

- fast, convenient online submission

- thorough peer review by experienced researchers in your field

- rapid publication on acceptance

- support for research data, including large and complex data types

- gold Open Access which fosters wider collaboration and increased citations

- maximum visibility for your research: over $100 \mathrm{M}$ website views per year

At BMC, research is always in progress.

Learn more biomedcentral.com/submissions 